\title{
Developing a complex intervention for diet and activity behaviour change in obese pregnant women (the UPBEAT trial); assessment of behavioural change and process evaluation in a pilot randomised controlled trial
}

Lucilla Poston ${ }^{1 *}$, Annette L Briley ${ }^{1}$, Suzanne Barr ${ }^{2}$, Ruth Bell ${ }^{3}$, Helen Croker ${ }^{6}$, Kirstie Coxon ${ }^{1}$, Holly N Essex ${ }^{5}$, Claire Hunt ${ }^{1}$, Louise Hayes ${ }^{3}$, Louise M Howard', Nina Khazaezadeh', Tarja Kinnunen ${ }^{8}$, Scott M Nelson?', Eugene Oteng-Ntim ${ }^{1}$, Stephen C Robson ${ }^{4}$, Naveed Sattar ${ }^{7}$, Paul T Seed ${ }^{1}$, Jane Wardle ${ }^{6}$, Thomas AB Sanders ${ }^{2}$ and Jane Sandall ${ }^{1}$

\begin{abstract}
Background: Complex interventions in obese pregnant women should be theoretically based, feasible and shown to demonstrate anticipated behavioural change prior to inception of large randomised controlled trials (RCTs). The aim was to determine if a) a complex intervention in obese pregnant women leads to anticipated changes in diet and physical activity behaviours, and b) to refine the intervention protocol through process evaluation of intervention fidelity.

Methods: We undertook a pilot RCT of a complex intervention in obese pregnant women, comparing routine antenatal care with an intervention to reduce dietary glycaemic load and saturated fat intake, and increase physical activity. Subjects included 183 obese pregnant women (mean BMl $36.3 \mathrm{~kg} / \mathrm{m}^{2}$ ).

Diet was assessed by repeated triple pass 24-hour dietary recall and physical activity by accelerometry and questionnaire, at $16^{+0}$ to $18^{+6}$ and at $27^{+0}$ to $28^{+6}$ weeks gestation in women in control and intervention arms. Attitudes to behaviour change and quality of life were assessed and a process evaluation undertaken. The full RCT protocol was undertaken to assess feasibility.

Results: Compared to women in the control arm, women in the intervention arm had a significant reduction in dietary glycaemic load (33 points, $95 \% \mathrm{Cl}-47$ to -20$),(\mathrm{p}<0.001)$ and saturated fat intake $(-1.6 \%$ energy, $95 \% \mathrm{Cl}$ -2.8 to -0.3$)$ at 28 weeks' gestation. Objectively measured physical activity did not change. Physical discomfort and sustained barriers to physical activity were common at 28 weeks' gestation. Process evaluation identified barriers to recruitment, group attendance and compliance, leading to modification of intervention delivery.

(Continued on next page)
\end{abstract}

\footnotetext{
* Correspondence: lucilla.2.poston@kcl.ac.uk

'Division of Women's Health, Women's Health Academic Centre, King's College London and King's Health Partners, 10th floor, North Wing, St. Thomas' Hospital, London SE1 7EH, UK

Full list of author information is available at the end of the article
} 
(Continued from previous page)

Conclusions: This pilot trial of a complex intervention in obese pregnant women suggests greater potential for change in dietary intake than for change in physical activity, and through process evaluation illustrates the considerable advantage of performing an exploratory trial of a complex intervention in obese pregnant women before undertaking a large RCT.

Trial registration: Trial Registration Number: ISRCTN89971375

Keywords: Pregnancy, Obesity, Diet, Physical activity, Complex intervention, Evaluation

\section{Background}

Obesity is prevalent in women of reproductive age in both high and low-to-middle income countries [1]. Pregnant obese women have a heightened risk of adverse pregnancy outcomes [2], but at present there is no evidence-based intervention that can be introduced into clinical practice to improve pregnancy outcome in obese women. The majority of attempts to develop interventions have hitherto focused on limiting gestational weight gain (GWG) according to the USA Institute of Medicine (IOM) recommendations [3]. Recent meta-analyses of relevant studies in obese women show modest restriction of GWG without robust evidence for improved clinical outcome [4,5]. Limitations of the existing evidence include poor study design, small sample size, absence of a theoretical basis and, importantly, no a priori demonstration of the feasibility of the intervention in regard to changing the specific behaviours targeted [6]. We have developed a theoretically based behavioural group intervention (diet and physical activity) for obese pregnant women with the primary aim of improving maternal glucose homeostasis. As maternal insulin resistance is integral to many complications of obese pregnancy the dietary intervention focuses on lowering the dietary glycaemic index (GI), previously shown to improve pregnancy outcome in women with gestational diabetes (GDM) $[7,8]$. Increased physical activity can also improve metabolic control and reduce GDM risk in pregnant women [9].

Prior to embarking on a large randomised controlled trial (RCT) and in accordance with UK Medical Research Council Guidance for development of a complex intervention [10], we first explored the theoretical basis for an intervention in obese pregnant women $[5,6,11,12]$, leading to development of a novel intervention (Phase 1). We now report on Phase 2, an exploratory trial to determine whether this intervention achieved the changes in dietary and physical activity behaviours anticipated, and to undertake a process evaluation of every aspect of fidelity of the intervention and the protocol.

\section{Subjects}

Potentially eligible participants attending clinics for general antenatal care were approached by research midwives in four UK study centres in urban settings providing a range of models of care. The contributing hospitals were 1) The Southern General Hospital and Princess Royal Maternity Hospital (Glasgow), 2) The Royal Victoria Infirmary (Newcastle), 3) Guy's and St Thomas' NHS Foundation Trust (London) and 4) King's College Hospital Foundation Trust (London).

\section{Methods}

The protocol for the exploratory trial is shown in Figure 1.

Verbal and printed information was provided to potential participants at a routine antenatal appointment in the first trimester and women were contacted $>24$ hrs later to ascertain willingness to participate. For those declining participation, consent to record basic demographic data and BMI were obtained. Those willing to participate were invited to return for their first study appointment in the early second trimester $\left(>15^{+0}\right.$ weeks to $<17^{+6}$ weeks' gestation). This window of recruitment allowed adequate time for arrangement of the one to one session with the health trainer followed by the eight week intervention programme prior to the oral glucose tolerance test, carried out between $27^{+0}$ and $28^{+6}$ weeks' gestation. Research midwives received a study-specific manual, attended at least one training session with the trial manager and continued feedback and training sessions for the study duration.

Inclusion criteria: $\mathrm{BMI} \geq 30 \mathrm{~kg} / \mathrm{m}^{2}$ and singleton pregnancy; gestational age $>15^{+0}$ weeks and $<17^{+6}$ weeks' gestation.

Exclusion Criteria: Unable or unwilling to give written informed consent; gestation $<15^{+0}$ weeks and $>17^{+6}$ weeks; pre-existing diabetes; pre-existing essential hypertension (treated); pre-existing renal disease; multiple pregnancy; systemic lupus erythematosus (SLE); antiphospholipid syndrome; sickle cell disease; thalassemia; celiac disease; currently prescribed metformin; thyroid disease or current psychosis.

All data were entered onto a password protected secure database (MedSciNet Ltd). Randomisation was performed online. The randomised treatment was allocated automatically, balanced by minimisation for maternal age, centre, ethnicity, parity and BMI. Data were analysed using Stata (version 11.2, StataCorp, College Station, Texas). All women randomised between 29th March 2010 and 13th 


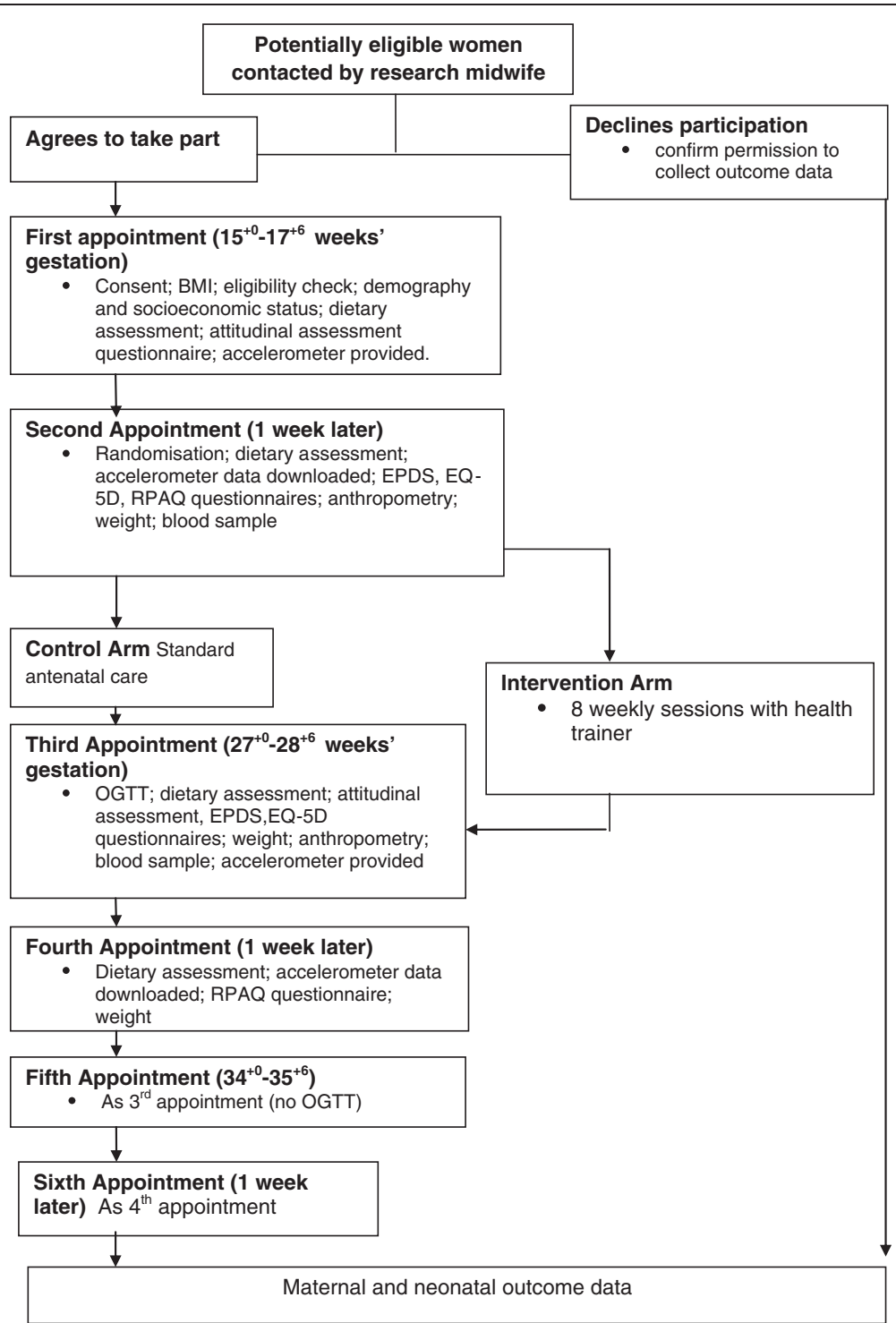

Figure 1 Study protocol. Abbreviations: BMI, Body Mass Index; EQ-5D, EuroQuol Quality of Life Questionnaire; EPDS, Edinburgh postnatal depression score questionnaire; OGTT, oral glucose tolerance test; RPAQ, Recent Physical Activity Questionnaire.

May 2011 were included. Postcodes were matched to two national indices of deprivation: the Index of Multiple Deprivation (IMD) for English addresses, or the Scottish Index of Multiple Deprivation (SIMD) for addresses in Scotland $[13,14]$.

\section{Control arm; standard care}

Following randomisation, women in the control arm returned for data collection appointments with the study midwife at $27^{+0}-28^{+6}$ and $34^{+0}-36^{+6}$ weeks', where possible coinciding with routine antenatal visits.

\section{Intervention arm}

Following randomisation, participants attended a oneto-one appointment with the health trainer $(\mathrm{HT})$ and were invited to weekly group sessions for 8 consecutive weeks from approximately 19 weeks' gestation.

All women attended routine antenatal care appointments and received advice regarding diet and physical activity (PA) in accordance with local policies, which draw on UK NICE guidelines [15].

\section{Sample size}

The primary outcome was change in dietary and PA behaviours at 28 weeks' gestation (coinciding with the primary maternal outcome for the main RCT, GDM at 28 weeks'). No prior investigation in obese pregnant women was available to inform power at the planning stage. The sample size of 183 was determined by the predefined duration of Phase 2, the exploratory phase. 
This number was adequate to enable power calculations for primary endpoints of the subsequent RCT, by providing estimates of the variance to within approximately $7 \%$ of the true value.

\section{Ethics}

Research Ethics Committee approval was obtained in all participating centres, UK Integrated Research Application System; reference 09/H0802/5 (South East London Research Ethics Committee).

\section{The intervention}

The intervention was informed by psychological models of health behaviour including control theory [16] and social cognitive theory [17]. Although no clear patterns between intervention characteristics and outcomes have been seen to date in lifestyle interventions in pregnancy, and few studies have described their theoretical basis [5,6]. Selfregulation techniques, drawn from control theory, suggest that behaviour change is facilitated by feedback about performance compared to pre-specified goals [16,18]. This approach was utilised in this study by setting 'SMART' (Specific, Measurable, Achievable, Relevant, and Time Specific) diet and activity goals, with behaviours recorded in a log book. Identification of benefits and overcoming barriers to behaviour change, and increasing self-efficacy were also included, and social support facilitated through the group format [17]. Following initial feedback from HTs regarding difficulties encountered by some women in attending sessions, for those women unable to attend, the session content was delivered by phone or email.

\section{Dietary advice}

Pre-specified dietary outcomes were a change in GI, glycaemic load (GL) (an indicator of carbohydrate quality (GI) and quantity consumed), and energy intake from saturated fatty acids (SFA). The focus of the dietary advice to the intervention group was therefore on increased consumption of foods with a low dietary GI, including replacement of sugar sweetened beverages with low GI alternatives. Reduction in saturated fats and replacement with monounsaturated and polyunsaturated fat was also recommended. Exchange of foods was emphasised e.g. a high GI food for a low GI food, rather than limiting energy intake.

\section{Physical activity advice}

Women in the intervention arm were encouraged to increase daily PA incrementally, setting goals of incremental step counts (monitored by pedometer) and maintaining the achieved PA level after the intervention period. Recommendations included an emphasis on walking at a moderate intensity level [19].

\section{Intervention delivery}

The intervention was delivered by health trainers (HTs). In the UK, HTs do not have pre-specified health professional qualifications, but relevant experience (http:// informationstrategy.dh.gov.uk/health-trainer-workforce). All HTs received a comprehensive treatment manual, prestudy training (and within-study supervision) in behaviour modification and conducting group sessions (organised by Weight Concern; Registered Charity 1059686). The sessions were held in a hospital setting in all but one centre, where women attended a community children's centre. At the initial one-to-one appointment women were provided with a participant handbook, reflecting the rationale and content of the HT sessions, a pedometer (Yamax SW-200 Digiwalker), a log book for weekly SMART goals and related behaviours (steps, PA and diet) and a DVD of a specially devised pregnancy exercise regime. Potential benefits of attending group sessions were discussed. Each group session delivered a different element of the dietary and PA intervention. Additional file 1: Table S1. Goals from the previous week were reviewed and goals set for the following week. Discussion included barriers to behavioural change and ways these might be overcome.

The following information was obtained from all participants (at visits indicated in Figure 1).

\section{Attitudinal assessment questionnaire}

The attitudinal assessment included questions relating to perceived benefits and barriers and confidence to carry out the dietary and PA behaviours [20,21]. The target behaviours were to consume lower GI carbohydrates, to reduce saturated fat intake and to increase PA.

\section{Health status and mental health}

The EuroQol quality of life (EQ- 5D) questionnaire [22] was used to assess health status, and the Edinburgh Post Natal Depression Score (EPDS) to assess mental health [23].

\section{Dietary assessment}

Repeated, triple pass $24 \mathrm{hr}$ recall data obtained at baseline (randomisation) and 28 weeks' gestation were evaluated twice, one week apart in both the intervention and control group. The $24 \mathrm{hr}$ dietary recall is a standard retrospective, interviewer led dietary assessment methodology used to capture information on all food and drinks consumed in the preceding $24 \mathrm{hrs}$. This is carried out in three stages (the triple pass), which includes 1) recording a 'quick' list of foods eaten or drunk, 2) collecting more detailed information of these foods and 3) reviewing all items once more in order to clarify any ambiguities or omissions. A short food frequency questionnaire (FFQ), for later validation, was also completed. 


\section{Physical activity assessment}

At the first and third appointments participants were asked to wear an Actigraph ${ }^{\mathrm{\tau}}$ accelerometer (Florida, USA) (either GT1M or GT3X set to uniaxial mode) for seven consecutive days, removing it for washing, bathing, swimming and at night. PA was also assessed by questionnaire (Recent Physical Activity Questionnaire (RPAQ).

\section{Process evaluation}

A process evaluation, following Steckler and Linnan's [24] framework was undertaken. This explored 1) Context (environmental, socio-economic or political factors), 2) Reach (the proportion of the intended target audience that participates, and which subgroups, if any, do not participate), 3) Dose delivered and dose received (the proportion of intended intervention received) 4) Fidelity (if each component of the complex intervention was provided as intended) and 5) Acceptability (if the intervention materials and advice were well received by providers and participants).

Qualitative semi-structured interviews were conducted, to capture women's experiences and perceptions of the trial and intervention. Women were recruited from each of the participating study sites using a maximum diversity sampling approach, following an informed consent procedure. Interviews took place between November 2010 and February 2011, and were either face-to-face $(n=17)$, mostly in hospital settings, or by phone $(n=4)$. Control $(n=12)$ and intervention $(n=9)$ interviewees were asked about their involvement in the research and their experiences of the trial appointments, measurements, blood tests and accelerometry recordings. Women in the intervention arm were additionally asked about their perceptions of the different components of the intervention, and how these impacted upon their lives. The interviews were conducted by one researcher and took place during pregnancy after the intervention had been provided. In addition, health trainers completed audio diaries (130 recordings) in which they reflected on the fidelity and feasibility of the intervention delivery. Attendance at sessions was recorded on the study database.

\section{Clinical outcome data}

Maternal primary outcome for the subsequent RCT (diagnosis of GDM)

A blood sample for fasting glucose and insulin was taken after an overnight fast. For the OGTT, following a glucose load (410 $\mathrm{ml}$ of lucozade or $75 \mathrm{~g}$ glucose in water), $1 \mathrm{hr}$ and $2 \mathrm{hr}$ samples were taken for glucose measurement. Diagnosis of GDM was confirmed by fasting glucose $\geq 5.1 \mathrm{mmol} / \mathrm{L}$ and/or $1 \mathrm{hr}$ glucose $\geq 10 \mathrm{mmol} / \mathrm{L}$; $2 \mathrm{hr}$ glucose $\geq 8.5 \mathrm{mmol} / \mathrm{L}$ according to the International
Association of the Diabetes and Pregnancy Study Groups (IADPSG) guidelines [25]. Following GDM diagnosis, women were referred for routine GDM care according to local criteria.

\section{Neonatal primary outcome for the subsequent RCT (large for gestational age delivery (LGA) defined as $>90^{\text {th }}$ customised birthweight centile)}

Customised birthweight centiles were calculated correcting for gestational age, maternal ethnicity, weight and height in early pregnancy, parity and infant sex [26]. Weight adjustment for women with $\mathrm{BMI} \geq 30 \mathrm{~kg} / \mathrm{m}^{2}$ is based on a notional weight corresponding to a BMI of $29.9 \mathrm{~kg} / \mathrm{m}^{2}$.

\section{Outcome data also recorded (not reported)}

These included maternal outcomes: diagnosis of GDM and pre-eclampsia, gestational weight gain, mode of delivery, blood loss at delivery, inpatient nights, detailed clinical and family history, health in current pregnancy, early pregnancy data (ultrasound scan, nuchal screening), blood pressure, routine blood results; neonatal outcomes: gestational age at delivery, birthweight, anthropometry, inpatient nights. Maternal urine and cord blood samples were also provided.

\section{Data handling and statistical analysis Health quality and attitudinal assessment questionnaires} The generic EQ-5D health-related quality of life instrument [22] is reported as the proportion of women with problems on individual dimensions (mobility, self-care, usual activities, pain/discomfort, and anxiety/depression). It is given as a summary index score calculated from preference values of different combinations of the dimensions elicited using the time trade-off method in a sample representative of non-institutionalized adults in England, Scotland and Wales (range -0.59 to 100 where -0.59 is severe problems on all dimensions) $[27,28]$; and also the visual analogue scale (VAS) of health-related quality of life (range 0 to 100 where 0 is worst imaginable health state). The change between baseline and 28 weeks' gestation in the percentage of women with any problem was assessed using McNemar's test of changes. Attitudes to target behaviours (attitudinal assessment questionnaire) are based on the average of multiple responses on 5-point scales (3 responses for diet, 13 for PA) with 5 indicating the greatest perceived barrier, perceived benefit, or level of confidence.

\section{Assessment of deprivation}

These scales for estimation of deprivation in England and Scotland $[13,14]$ use different reference populations to determine the actual indices of deprivation, and are therefore not directly comparable. For the purposes of this study, the most deprived quintile is presented 
separately for women in each population and compared to the remainder of the population (quintiles 1-4).

\section{Dietary analysis}

Quality of dietary data was checked within one week of entry. Dietary coding utilised McCance and Widdowson "Composition of Foods" ( $6^{\text {th }}$ edition) food codes and nutrient composition was evaluated using WISP 3.0 (Tinuviel Software) for GI and GL values [29]. Estimates using previously published methodology were made when GI values were not available [30]. $24 \mathrm{hr}$ recall data obtained at baseline (randomisation) and at 28 weeks' gestation was evaluated twice, one week apart, and data were averaged. The validity of the short FFQ was assessed against the dietary recall data. Pre-specified dietary outcomes were a change in GI, GL and energy intake from SFA. Total energy intake, the proportion of energy derived from macronutrients were assessed.

\section{Actigraph analysis}

An epoch length (time sampling interval) of 15 seconds was specified. Data were processed using the MAHUFFE Software package [31]. Sedentary behaviour was defined as $<100$ counts per minute (cpm), light activity as 100$1951 \mathrm{cpm}$, moderate intensity activity as $1952-5725 \mathrm{cpm}$ and vigorous activity as $>5725 \mathrm{cpm}$ [32]. As time spent in vigorous activity was very low, minutes of moderate and vigorous physical activity (MVPA) were combined. Runs of zero counts lasting $>60$ minutes were excluded, as these indicated monitor removal. A valid recording was defined as a day in which $>500$ minutes of monitored on-time were recorded in $24 \mathrm{hrs}$ [33]. Data from participants recording $\geq 3$ days of valid accelerometry data were included in the analysis. The specified PA outcome was an increase in minutes per day of MVPA recorded by accelerometry.

\section{Recent physical activity questionnaire (RPAQ)}

The RPAQ was modified for the assessment of PA in the preceding seven days. Estimates of minutes per day spent in light, moderate and vigorous activity in each of the domains were calculated. Sedentary activities were defined as those with a metabolic equivalent (MET) of $<1.5$. Light activities were those of 1.5 to 3 METs. Moderate activities were those of 3 to 6 METs. Vigorous activities were those of 6 METs or greater [34] MVPA were combined to give one summary variable.

\section{Process evaluation}

Interviews were recorded and transcribed verbatim. Transcripts were anonymised and a unique identifier (ID number) was used to maintain confidentiality. Data were imported into a qualitative software analysis package (NVivo 8), and subject to comparative thematic analysis [35]. To enhance study validity and reliability, themes arising from the research were discussed, the data supporting these was reviewed by co-researchers, and data were compared between sites and with existing literature. By these methods, assumptions were tested and observations of differences and their relationship to the theoretical models underpinning the study were explored.

\section{Statistical analysis}

Analyses followed the intention-to-treat principle. Following CONSORT guidelines, risk ratios and risk differences were estimated by binary regression for Yes/No outcomes. Where measures were repeated at baseline and 28 weeks' gestation, results [mean (SD) n (\%)] are presented separately at each time point. Randomised comparisons at 28 weeks' were made using linear regression with robust standard errors, adjusting for the baseline value. For PA data, dummy variables were used when the baseline values were missing. Correlations between PA as assessed objectively (accelerometry) and when self-reported (RPAQ) were explored.

\section{Results}

Figure 2 provides a flow chart of participants through the study.

\section{Participants}

Mean first visit BMI was $36.3 \mathrm{~kg} / \mathrm{m}^{2}$. More than half the women were White and the remainder from Black (38\%) and Minority Ethnic communities. More than half (56\%) already had at least one child. More than half of those from centres in England and over 40\% in Scotland came from regions in the highest quintile of social deprivation (Table 1).

\section{Diet}

Table 2 shows the dietary intakes at baseline and at 28 weeks of gestation. There were no differences between groups in energy intakes, GI, GL, or other macronutrient at baseline. However, following the intervention, at 28 weeks' gestation, total energy intake, dietary GL, GL (\%E), saturated fat (\%E) and total fat $(\% \mathrm{E})$, were significantly lower and fibre intake measured as non-starch polysaccharides was greater in the intervention group than in the control arm. The proportion of energy derived from protein was higher in the intervention group but absolute protein intake did not differ. There was a difference of 7 GI points between the intervention and control group which achieved borderline statistical significance $(P=0.054)$.

\section{Physical activity}

There were no differences between the intervention and control arms in objectively measured PA variables at baseline or at 28 weeks' gestation, after adjustment for baseline activity. Self-reported moderate to vigorous PA 


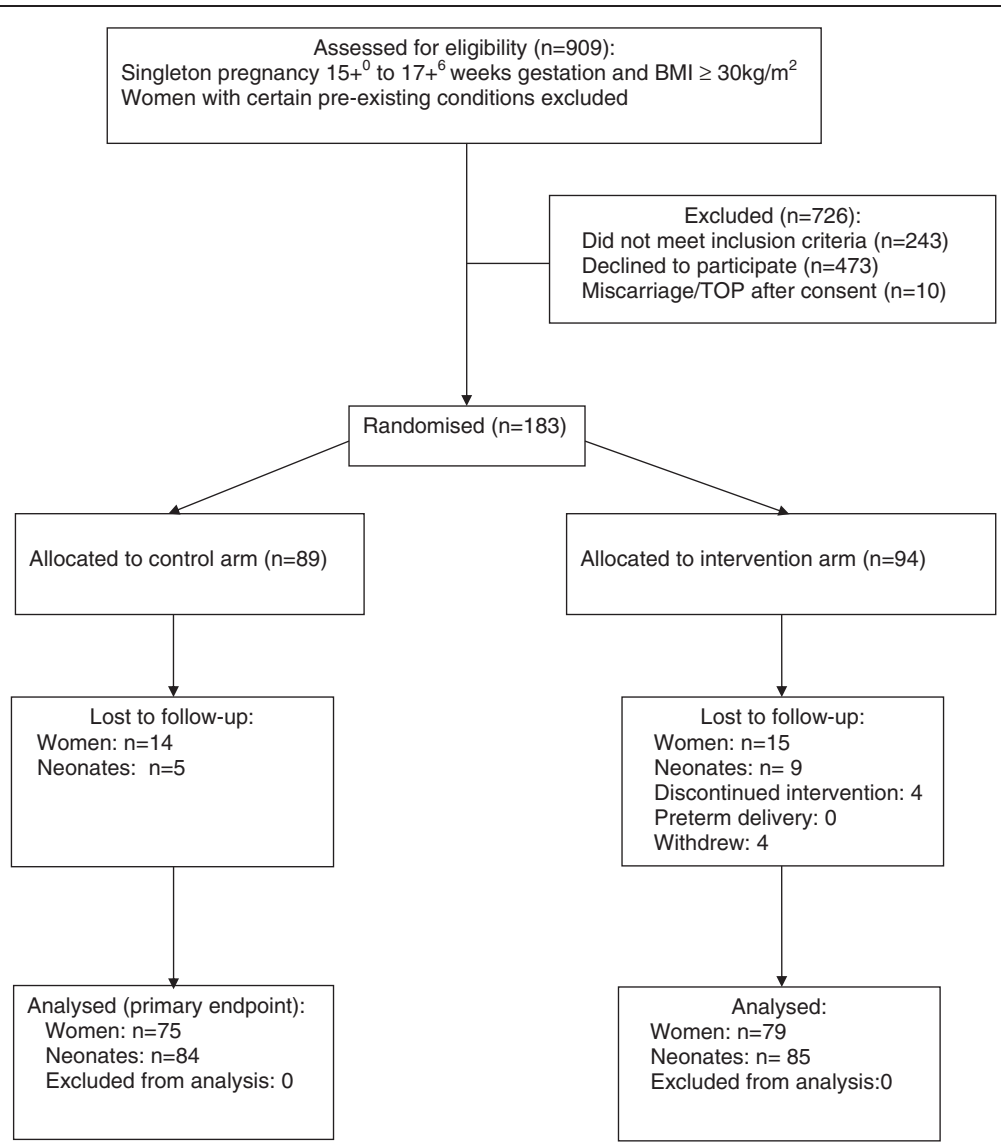

Figure 2 Consort Diagram. Flow chart of participants through study.

(MVPA) at 28 weeks' gestation was increased in the intervention group (mean difference 34 minutes/day; 95\% CI 9 to $59 \mathrm{~min} /$ day), but this was not supported by the objective data. Women in the intervention group self-reported walking for leisure for $14 \mathrm{~min} /$ day more than those in the control group at 28 weeks' gestation (95\% CI 5 to $23 \mathrm{~min}, \mathrm{p}=0.003$ ). Agreement between the RPAQ questionnaire and accelerometry was very poor, for example, correlations between MVPA in the two formats at baseline were $\mathrm{r}=0.275$ (95\% CI: 0.107 to 0.428 ) and at 28 weeks, $\mathrm{r}=-0.069$ (95\% CI: -0.296 to 0.165$)$ Table 3.

\section{Attitudinal assessment of target behaviours}

Benefits, barriers and confidence in making the target physical activity and dietary changes were unchanged in either the control and intervention groups from baseline to 28 weeks gestation Table 4 .

\section{Health Status and Mental Health EPDS EQ-5D}

There was no influence of the intervention on the numbers of women reporting problems in each of the EQ-5D domains, but as a group, obese women experienced a significant increase in problems with mobility, self care, usual activities and pain and discomfort from baseline to
28 weeks' gestation. There was a $10 \%$ prevalence of probable depression at baseline and $13 \%$ at 28 weeks (i.e. EPDS score>12) with no significant effect of the intervention on anxiety and depression at 28 weeks Table 4.

\section{Process evaluation \\ Context}

This study coincided with publication of new reports and guidance for obesity in pregnancy with associated media coverage [15,36]. Most control group interviewees demonstrated awareness and reported taking steps to improve their diet or fitness. Additional file 1: Table S2.

\section{Reach}

Those approached who were eligible for recruitment but declined to participate $(n=473)$ were of mean age 29.9 years; mean BMI $35.39 \mathrm{~kg} / \mathrm{m}^{2}$; ethnicity, $59.7 \%$ White, $32.8 \%$ Black and $43.0 \%$ were in the lowest quintile for Index of deprivation indicating the most severe deprivation. Characteristics of participants providing semi-structured interviews $(n=21)$ are shown in Additional file 1: Table S3. This demographic profile was similar to study participants (Table 1). Overall, 29/183 (15.8\%) women were lost to follow up Figure 2. 
Table 1 Description of subjects at baseline $\left(16^{+0}-18^{+6}\right.$ weeks gestation) by randomised treatment

\begin{tabular}{lll}
\hline Control & Intervention \\
\hline$n=89$ & $n=94$
\end{tabular}

\begin{tabular}{|c|c|c|}
\hline Age (years) ${ }^{1}$ & $30.7(4.9)$ & $30.4(5.7)$ \\
\hline \multicolumn{3}{|c|}{ Age categories } \\
\hline $18-25$ & $16(18 \%)$ & $22(23 \%)$ \\
\hline $26-30$ & $25(28 \%)$ & $27(29 \%)$ \\
\hline $31-40$ & $46(52 \%)$ & $42(45 \%)$ \\
\hline 41 plus & $2(2 \%)$ & $3(3 \%)$ \\
\hline \multicolumn{3}{|c|}{ Anthropometry } \\
\hline Height (m) & $1.64(0.07)$ & $1.64(0.07)$ \\
\hline Weight (kg) & $96.8(16.2)$ & $97.8(12.7)$ \\
\hline $\mathrm{BMI}\left(\mathrm{kg} / \mathrm{m}^{2}\right)$ & $36.1(4.8)$ & $36.5(4.7)$ \\
\hline \multicolumn{3}{|l|}{ Ethnicity $^{1}$} \\
\hline White & $51(57 \%)$ & $52(55 \%)$ \\
\hline Black & $32(36 \%)$ & $38(40 \%)$ \\
\hline Asian & $1(1 \%)$ & $2(2 \%)$ \\
\hline Other & $5(6 \%)$ & $2(2 \%)$ \\
\hline \multicolumn{3}{|l|}{ Parity $^{1}$} \\
\hline 0 & $38(43 \%)$ & $42(45 \%)$ \\
\hline 1 & $36(40 \%)$ & $29(31 \%)$ \\
\hline 2 or more & $15(17 \%)$ & $23(24 \%)$ \\
\hline \multicolumn{3}{|c|}{ Cigarette smoking } \\
\hline Never & $61(68 \%)$ & $63(67 \%)$ \\
\hline Ex-smoker & $22(25 \%)$ & $25(27 \%)$ \\
\hline Current & $6(7 \%)$ & $6(6 \%)$ \\
\hline
\end{tabular}

Number of cigarettes

1-5 per day

11-20 per day

6-10 per day

Index of multiple deprivation ${ }^{2}$

England

Mean (SD)

Quintiles

1-4 (less deprived)

5 (most deprived)

Scotland

Mean (SD)

Quintiles

1-4 (less deprived)

5 (most deprived)

Living arrangements

Single

With partner

With parent(s)

$\begin{array}{ll}83(93 \%) & 83(88 \%) \\ 3(3 \%) & 3(3 \%) \\ 1(1 \%) & 6(6 \%) \\ 2(2 \%) & 2(2 \%) \\ n=76 & n=79 \\ 34(12) & 36(14)\end{array}$

$35(46 \%)$

$29(37 \%)$

$41(54 \%)$

$50(63 \%)$

$\mathrm{n}=12$

$\mathrm{n}=14$

$28(11)$

$30(20)$

$7(58 \%)$

$8(57 \%)$

$5(42 \%)$

$6(43 \%)$

$35(39 \%)$

$50(53 \%)$

$66(74 \%)$

$69(73 \%)$

$7(8 \%)$
Table 1 Description of subjects at baseline $\left(16^{+0}-18^{+6}\right.$ weeks gestation) by randomised treatment (Continued)

\begin{tabular}{lll}
\hline Without partner or parents & $17(19 \%)$ & $17(18 \%)$ \\
Accommodation & & \\
Owned & $27(30 \%)$ & $21(22 \%)$ \\
Rented (private) & $26(29 \%)$ & $27(29 \%)$ \\
Rented (council) & $36(40 \%)$ & $46(49 \%)$ \\
\hline
\end{tabular}

Results shown are mean (SD) or $\mathrm{n}(\%)$.

${ }^{1}$ The randomised treatment allocation is balanced by minimization on maternal age, centre, ethnicity, and parity.

${ }^{2}$ The index of multiple deprivation is calculated for the region of residence

(Lower super output area in England, data region in Scotland) $[13,14]$.

Different methods and reference populations are used in England and

Scotland, and the indices are not directly comparable.

\section{Dose}

Of the 94 women randomised to the intervention, 82 (88\%) attended at least one group session, and 60 (64\%) attended 4 or more. A total of 42 women (45\%) received material from all eight sessions, 6 by full attendance (6\%) and the remainder when partly/wholly covered by subsequent phone contact. For all women, 6.1 (SD 2.6) sessions were attended or partly/wholly covered.

\section{Fidelity}

The intervention package (8 HT group sessions) was provided with good consistency at each study site. Goals were set at all group sessions, of which $88 \%$ were considered SMART by HTs according to their diaries. The maximum group size was 5 (mean 2 ).

\section{Acceptability}

Women in both arms of the trial found the research processes acceptable, and felt supported by the study midwives. Women in the intervention group were generally willing, in principle, to attend the eight health trainer sessions, and most women who attended valued the group approach, citing opportunities to raise questions and discuss each other's experiences. Some were surprised at the extent of the intervention, having anticipated a less intensive, more advice-based approach.

Consistency of attendance at the HT sessions varied for different reasons including work commitments, school pick-up times, or feeling too unwell or tired. Occasionally initial involvement waned when groups proved smaller than anticipated, although the HT input by phone or email was considered valuable.

Some women found the information contained in the handbook new, whilst for others it was too basic. The pedometers and step goals were generally well received. Setting and reflecting on weekly goals was motivational for most, but could also invoke feelings of guilt, or a sense of being observed and judged. Women reported having watched the DVD, but few used it regularly. 
Table 2 Dietary outcomes

\begin{tabular}{|c|c|c|c|c|c|}
\hline & & Control & Intervention & Difference $(95 \%$ CI) & $P$ value \\
\hline n: baseline, 28 weeks' & & $n=89,69$ & $n=94,71$ & & \\
\hline \multirow[t]{2}{*}{ Total Energy (MJ/d) } & Baseline & $7.53(2.21)$ & $7.26(2.29)$ & & \\
\hline & 28 weeks & $7.71(2.30)$ & $6.75(2.57)$ & $-0.94(-1.72$ to -0.18$)$ & 0.016 \\
\hline \multirow[t]{2}{*}{ Dietary GI (\%) } & Baseline & $58(6)$ & $58(5)$ & & \\
\hline & 28 weeks & $60(26)$ & $53(13)$ & -7 ( -15 to 0$)$ & 0.054 \\
\hline \multirow[t]{2}{*}{ Dietary GL (g/d) } & Baseline & $133(48)$ & $129(41)$ & & \\
\hline & 28 weeks & $146(55)$ & $111(39)$ & $-33(-47$ to -20$)$ & $<0.001$ \\
\hline \multirow[t]{2}{*}{ GL (\%E) } & Baseline & $27.7(5.3)$ & $28.5(5.9)$ & & \\
\hline & 28 weeks & $31.3(13.3)$ & $26.6(8.0)$ & $-4.8(-8.5$ to -1.0$)$ & 0.013 \\
\hline \multirow[t]{2}{*}{ Carbohydrate (\%E) } & Baseline & $48.0(8.4)$ & $48.9(9.6)$ & & \\
\hline & 28 weeks & $48.2(8.0)$ & $50.0(8.2)$ & $1.7(-1.0$ to 4.4$)$ & 0.207 \\
\hline \multirow[t]{2}{*}{ Protein (\%E) } & Baseline & $15.5(3.6)$ & $16.0(4.2)$ & & \\
\hline & 28 weeks & $15.5(3.2)$ & $17.1(4.9)$ & 1.5 ( 0.1 to 2.8 ) & 0.034 \\
\hline \multirow[t]{2}{*}{ Protein (g) } & Baseline & $69.3(25.3)$ & $68.5(26.1)$ & & \\
\hline & 28 weeks & $70.6(24.0)$ & $66.5(23.5)$ & $-4.8(-12.3$ to 2.6$)$ & 0.204 \\
\hline \multirow[t]{2}{*}{ Total fat (\%E) } & Baseline & $36.0(8.2)$ & $34.9(9.3)$ & & \\
\hline & 28 weeks & $35.9(7.7)$ & $32.5(7.4)$ & $-3.2(-5.6$ to -0.8$)$ & 0.010 \\
\hline \multirow[t]{2}{*}{ SFA (\%E) } & Baseline & $12.7(3.9)$ & $12.0(4.3)$ & & \\
\hline & 28 weeks & $12.9(3.9)$ & $11.1(3.8)$ & $-1.6(-2.8$ to -0.3$)$ & 0.015 \\
\hline \multirow[t]{2}{*}{ MUFA (\%E) } & Baseline & $12.1(4.1)$ & $11.4(4.0)$ & & \\
\hline & 28 weeks & $11.6(4.0)$ & $10.4(3.2)$ & $-1.0(-2.2$ to 0.2$)$ & 0.088 \\
\hline \multirow[t]{2}{*}{ PUFA (\%E) } & Baseline & $6.4(3.0)$ & $6.0(3.1)$ & & \\
\hline & 28 weeks & $5.9(2.8)$ & $6.0(2.7)$ & $0.13(-0.8$ to 1.1$)$ & 0.774 \\
\hline \multirow[t]{2}{*}{ P:S ratio } & Baseline & $0.56(0.31)$ & $0.56(0.40)$ & & \\
\hline & 28 weeks & $0.51(0.35)$ & $0.64(0.52)$ & $0.13(-0.01$ to 0.28$)$ & 0.075 \\
\hline \multirow[t]{2}{*}{ NSP (g) } & Baseline & $11.2(4.6)$ & $10.4(4.6)$ & & \\
\hline & 28 weeks & $10.5(4.2)$ & $12.0(6.0)$ & 1.77 ( 0.08 to 3.47 ) & 0.040 \\
\hline
\end{tabular}

Abbreviations: Gl glycaemic index, GL glycaemic load, MUFA monounsaturated fatty acid, NSP non-starch polysaccharide, $P: S$ ratio polyunsaturated fatty acid, saturated fatty acid ratio; PUFA polyunsaturated fatty acid, SFA saturated fatty acids,\%E: percentage energy.

For each dietary variable, results are presented in two lines: at trial entry (Baseline; $16^{+0}-18^{+6}$ weeks' gestation) and after randomised treatment, with comparisons and p-values only for the randomised comparison. Comparisons are adjusted for baseline levels throughout.

When interviewees were asked whether they had made any changes as a result of the intervention, most reported some degree of change, especially in relation to dietary intake. Reported changes in PA were more limited, particularly due to pelvic pain or tiredness as pregnancy progressed. Women often reported aspirations to increase exercise postnatally. See Additional file 1: Table S3 and $\mathrm{S} 4$ for extracts of interviews.

\section{Maternal and neonatal outcomes}

The primary maternal and neonatal outcomes for the subsequent RCT are shown in Table 5. There were no significant differences in GDM or LGA $\left(\geq 90^{\text {th }}\right.$ customised centile) between control and intervention arms. There was also no significant difference in gestational weight gain between control and intervention arms (secondary outcome).

The overall incidence of GDM, the primary outcome of the subsequent RCT (not powered for), according to recent International Association of the Diabetes and Pregnancy Study Groups (IADPSG) criteria [25] was 30\%, enabling calculation of the subsequent RCT sample size (1546 women) for the RCT, powered for a $25 \%$ reduction. Since $38 \%$ of potentially eligible women took part in the pilot study, to achieve this sample size in the main RCT, approximately 4100 would need to be approached.

\section{Discussion}

This study describes a pragmatic and rigorously evaluated pilot study of a complex intervention for diet and 
Table 3 Physical activity as measured by accelerometer and RPAQ questionnaire

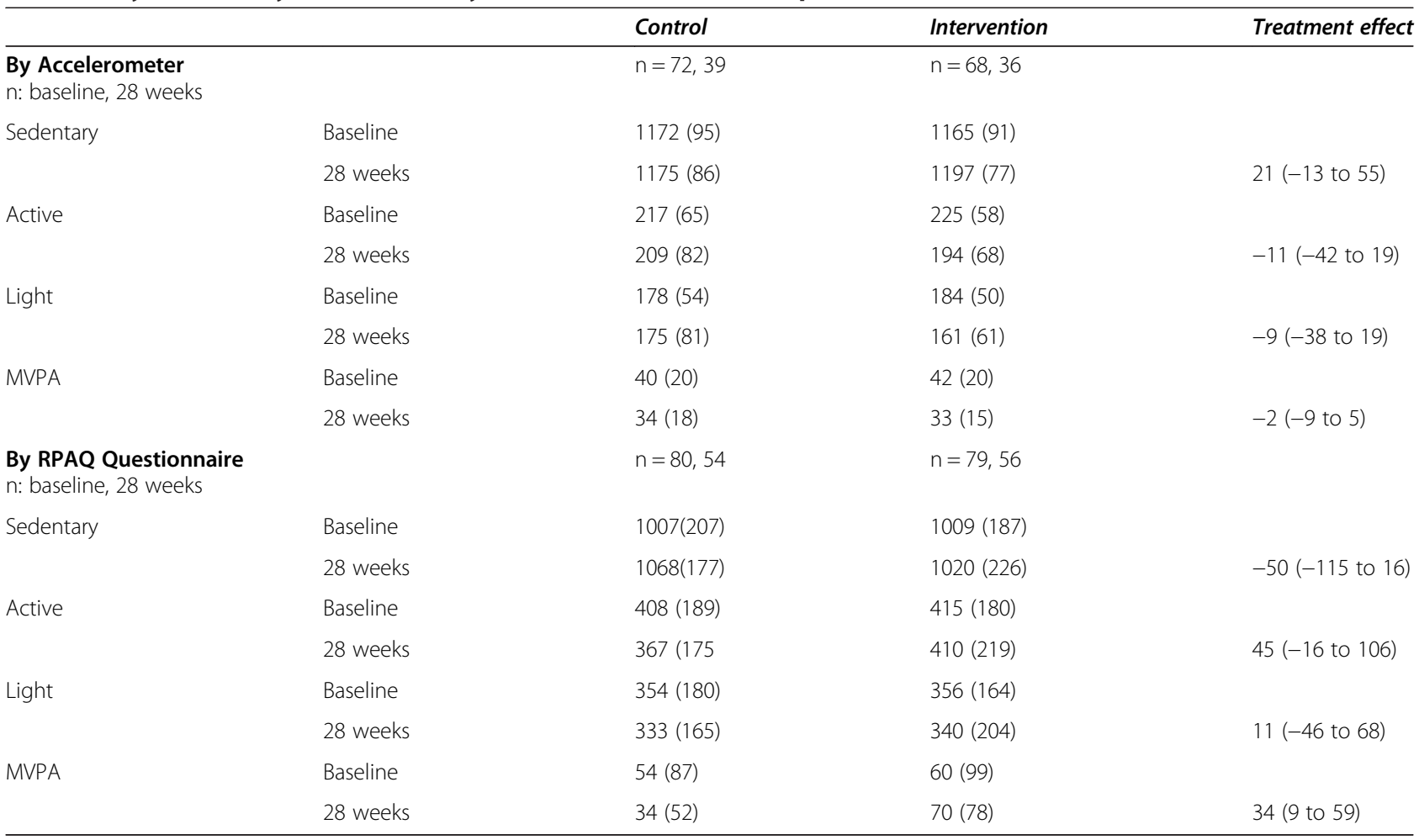

Abbreviations: MVPA, Moderate and/or vigorous physical activity; RPAQ, Recent Physical Activity Questionnaire.

Results are measured in minutes/day presented as mean (SD). Treatment effects are mean differences ( $95 \%$ confidence intervals), adjusted for baseline activity; with dummy variables where baseline levels missing (2 accelerometer, 3 RPAQ).

activity behaviour change in obese pregnant women. The intervention was associated with a significant change in dietary behaviour. Process evaluation showed overall acceptability of the protocol but led to several refinements to improve acceptability and fidelity.

In any lifestyle intervention of diet and physical activity, it is important that the pilot study design includes methods to assess the potential of the intervention to change these behaviours in the anticipated direction of effect. Few assessments of dietary intake in similar investigations of overweight or obese women have been attempted [37-39]. We used a $24 \mathrm{hr}$ recall method to assess dietary intake and while this may lead to under-reporting of energy intakes, the reported values are not dissimilar to those of nonpregnant women in general UK population [40]. The objectives of the dietary intervention, to bring about reductions in GL and the proportion of energy derived from saturated fatty acids, were both achieved. This suggests that obese pregnant women are amenable to changing their diet in response to an intervention based on established theory, and that dietary advice, frequently delivered by health professionals, is likely to be successful in achieving dietary change in obese pregnant women, as previously implied [38]. The reduction in dietary GL achieved was similar $33 \% \mathrm{v}$ $45 \%)$ to that reported in obese type 2 diabetic nonpregnant subjects in which improved glycaemic control was achieved [41]. Recently a similar intervention in 759 pregnant women, showed a lower change in GL (13\%), which was associated with a reduction in gestational weight gain in women who had previously delivered a large for gestational age infant [42].

The reduction in energy intake observed is consistent with other studies that have restricted the intake of fat from meat and dairy products which have not been replaced by other sources of food energy [43]. The reduced GL may also have contributed through effects on satiety [44]. To our knowledge this is the first study demonstrating that anticipated changes in diet occur following delivery of an intervention to lower GL and saturated fat in obese pregnant women without GDM. Importantly this occurred despite the focus being on reducing GL by lowering the intake of added sugars as well as advocating foods with a lower GI. Focusing on GI tends to modify the GL from starch whereas the GI from sugar sweetened beverages is less amenable to change. Consequently, dietary advice to decrease the intake of added sugar, particularly as sugar-sweetened beverages, is likely to have had an important impact on GL. 
Table 4 Attitudes to target behaviours, quality of life and mental health assessment

\begin{tabular}{|c|c|c|c|c|}
\hline Attitudes to target behaviours & & Control & Intervention & Treatment effect \\
\hline \multicolumn{5}{|l|}{ Barriers } \\
\hline \multirow[t]{2}{*}{ Diet } & Baseline & $2.49(0.58)$ & $2.37(0.61)$ & \\
\hline & 28 weeks & $2.45(0.58)$ & $2.14(0.68)$ & $-0.18(-0.35$ to 0.00$)$ \\
\hline \multirow[t]{2}{*}{ Physical activity } & Baseline & $2.64(0.55)$ & $2.48(0.63)$ & \\
\hline & 28 weeks & $2.47(0.50)$ & $2.20(0.61)$ & $-0.20(-0.37$ to -0.03$)$ \\
\hline \multicolumn{5}{|l|}{ Perceived benefits } \\
\hline \multirow[t]{2}{*}{ Diet } & Baseline & $3.75(0.72)$ & $3.80(0.64)$ & \\
\hline & 28 weeks & $3.79(0.67)$ & $3.97(0.80)$ & $0.13(-0.10$ to 0.36$)$ \\
\hline \multirow[t]{2}{*}{ Physical activity } & Baseline & $3.94(0.70)$ & $4.04(0.54)$ & \\
\hline & 28 weeks & $3.84(0.60)$ & $4.06(0.69)$ & $0.17(-0.04$ to 0.38$)$ \\
\hline \multicolumn{5}{|l|}{ Confidence } \\
\hline \multirow[t]{2}{*}{ Diet } & Baseline & $3.78(0.75)$ & $3.84(0.64)$ & \\
\hline & 28 weeks & $3.71(0.72)$ & $3.85(0.81)$ & $0.11(-0.15$ to 0.37$)$ \\
\hline \multirow[t]{2}{*}{ Physical activity } & Baseline & $3.76(0.88)$ & $3.92(0.81)$ & \\
\hline & 28 weeks & $3.77(0.88)$ & $3.81(1.06)$ & $-0.05(-0.40$ to 0.30$)$ \\
\hline \multicolumn{2}{|l|}{ Quality of life (EQ-5D) } & Control & Intervention & Treatment effect \\
\hline \multicolumn{2}{|l|}{ n: baseline, 28 weeks } & $\mathrm{n}=87,75$ & $\mathrm{n}=94,80$ & \\
\hline \multicolumn{5}{|l|}{ Numbers reporting problems } \\
\hline \multirow[t]{2}{*}{ Mobility } & Baseline & $10(11 \%)$ & $11(12 \%)$ & \\
\hline & 28 weeks & $21(28 \%)$ & $25(31 \%)$ & $4 \%(-10$ to 18$)$ \\
\hline \multicolumn{2}{|l|}{ Change (all women) } & & & $19 \%$ (11 to 27$)$ \\
\hline \multirow[t]{2}{*}{ Self-care } & Baseline & $1(1 \%)$ & $0(0 \%)$ & \\
\hline & 28 weeks & $3(4 \%)$ & $3(4 \%)$ & $-0.3 \%$ ( -6 to 6$)$ \\
\hline \multicolumn{2}{|l|}{ Change (all women) } & & & $4 \%$ (0 to 8$)$ \\
\hline \multirow[t]{2}{*}{ Usual activities } & Baseline & $16(18 \%)$ & $13(14 \%)$ & \\
\hline & 28 weeks & $26(34 \%)$ & $26(33 \%)$ & $-1 \%(-15$ to 12$)$ \\
\hline \multicolumn{2}{|l|}{ Change (all women) } & & & $16 \%$ ( 8 to 24 ) \\
\hline \multirow[t]{2}{*}{ Pain \& discomfort } & Baseline & 38 (43\%) & $34(36 \%)$ & \\
\hline & 28 weeks & $45(60 \%)$ & $54(67 \%)$ & $10 \%(-1$ to 22$)$ \\
\hline \multicolumn{2}{|l|}{ Change (all women) } & & & $25 \%$ (17 to 34$)$ \\
\hline \multirow[t]{2}{*}{ Anxiety \& depression } & Baseline & $22(25 \%)$ & $20(21 \%)$ & \\
\hline & 28 weeks & $11(15 \%)$ & 17 (21\%) & $5 \%(-4$ to 15$)$ \\
\hline \multicolumn{2}{|l|}{ Change (all women) } & & & $-6 \%(-14$ to 1$)$ \\
\hline \multirow[t]{2}{*}{ TTO score } & Baseline & $0.85(0.18)$ & $0.88(0.14)$ & \\
\hline & 28 weeks & $0.79(0.24)$ & $0.79(0.16)$ & $-0.03(-0.07$ to 0.02$)$ \\
\hline \multicolumn{2}{|l|}{ Change (all women) } & & & $-0.08(-0.10$ to -0.06$)$ \\
\hline VAS (0 to 100$)$ & Baseline & $76(20)$ & $76(21)$ & \\
\hline & 28 weeks & $75(21)$ & $78(21)$ & $4(-3$ to 10$)$ \\
\hline Change (all women) & & & & $-2(-6$ to 2$)$ \\
\hline EPDS & & & & \\
\hline Total & Baseline & $7.1(4.6)$ & $7.4(4.5)$ & \\
\hline & 28 weeks & $6.9(4.2)$ & $7.1(5.2)$ & $0.1(-1.1$ to 1.3$)$ \\
\hline Total Score > 9 & Baseline & $25(29 \%)$ & $28(30 \%)$ & \\
\hline & 28 weeks & 17 (23\%) & $21(26 \%)$ & $1 \%(-9$ to 11$)$ \\
\hline
\end{tabular}


Table 4 Attitudes to target behaviours, quality of life and mental health assessment (Continued)

\begin{tabular}{lllll}
\hline Total score $>12$ & Baseline & $9(10 \%)$ & $10(11 \%)$ & \\
& 28 weeks & $6(8 \%)$ & $14(18 \%)$ & $7 \%(-1 \%$ to 16$)$ \\
\hline
\end{tabular}

Attitude to target behaviours are based on the average of multiple responses on 5 -point scales, with 5 indicating the greatest barrier, perceived benefit, or level of confidence, and 1 the least.

Abbreviations: EQ-5D EuroQol 5 dimensional quality of life scale, $T$ TO Time Trade Off health state ratings calculated from standard values elicited using the time trade-off method. VAS visual analogue scale, EPDS Edinburgh Postnatal Depression Score. Summaries are $\mathrm{n}(\%)$ or mean (SD) as appropriate. For the EuroQoL subscales, the overall change over time is estimated as a risk difference, by McNemar's test of changes. Elsewhere, differences are calculated by linear or binomal regression as appropriate, adjusting for baseline values.

This study adds to the scant literature on the habitual diet of obese pregnant women. The macronutrient profile at randomisation was similar to that of women in the general population, with fibre (non-starch polysaccharide) intake below, and total sugars and saturated fat above recommended UK guidelines [45]. The overall energy intake and macronutrient profile accords with one previous report in obese pregnancy [38]. Because of the time required to rigorously assess diet using the $24 \mathrm{hr}$ recall method which, according to the process evaluation is likely to have influenced recruitment and compliance, a short food frequency questionnaire (5-10 minutes) was evaluated for use in the subsequent RCT.

The few studies that have attempted to measure changes in PA in intervention trials in pregnancy have generally relied on self-report, and results have been equivocal [38,46-49]. Accelerometry, the standard method of objective assessment used previously in observational studies in pregnancy [48], has, to our knowledge, only been employed in one relevant RCT, the FitFor2 study, a supervised exercise intervention in 121 overweight and obese women [50]. Consistent with Fitfor2, we found no effect of the intervention on PA using the Actigraph accelerometer, concurring with the reported absence of change in barriers to PA. The failure of accelerometry to mirror the increase in self-reported walking in the intervention group could reflect insufficient intensity of this activity, but also reporting bias [51] which is common in the reporting of low intensity activities, such as those frequently undertaken by pregnant women [52].
As reported elsewhere, compliance with accelerometry in pregnancy was an issue [53,54]. Nonetheless, $60 \%$ of obese pregnant women providing baseline accelerometry data met the current guidelines for PA in pregnancy (i.e. $>30$ minutes of MVPA per day). A similar level of activity has been observed in pregnant women (all BMIs) [54] and overweight and obese non-pregnant adults [55], but not previously amongst obese pregnant women. Levels of PA were similar to those we found previously among overweight and obese women [53], but substantially higher than those reported for non-pregnant women in the UK [56]. There is no consensus on change of MVPA over pregnancy [53,57-59]. In this study of obese women both groups reduced the level of objectively measured MVPA as pregnancy progressed.

This assessment has highlighted a critical need to evaluate PA behaviour objectively. We may otherwise have erroneously concluded in the following RCT that increased PA does not affect clinically relevant outcomes. Despite showing no increase in PA, we have not recommended that the RCT focuses on diet only [4], but rather that women continue to be encouraged to adhere to PA recommended in clinical guidelines.

Although there were no changes in attitudinal outcomes, women were generally positive about the recommended dietary and physical activity behaviours despite perceived barriers to change. Attitudinal data relating to diet were comparable to a population sample of pregnant women [12]. The intervention did not achieve any reduction in perceived barriers, but despite this, important dietary changes were achieved which may infer

Table 5 Maternal and neonatal primary outcomes

\begin{tabular}{|c|c|c|c|c|c|}
\hline & Control & Intervention & Comparison & Treatment effect & Pvalue \\
\hline Maternal & $n=75$ & $n=79$ & & $(95 \% \mathrm{Cl})$ & \\
\hline \multirow[t]{2}{*}{ GDM } & $24(32 \%)$ & $22(28 \%)$ & Risk difference & $-4 \%(-19$ to 13$)$ & 0.574 \\
\hline & & & Risk ratio & 0.87 (0.54 to 1.41$)$ & \\
\hline Neonatal & $\mathrm{n}=84$ & $n=86$ & & & \\
\hline \multirow[t]{2}{*}{ LGA } & $7(8 \%)$ & $7(8 \%)$ & Risk difference & $0 \%(-8$ to 8$)$ & 0.982 \\
\hline & & & Risk ratio & 0.99 (0.36 to 2.7 ) & \\
\hline$>4 \mathrm{Kg}$ & $16(19 \%)$ & $13(15 \%)$ & Risk difference & $-4 \%(-15$ to 8$)$ & 0.518 \\
\hline
\end{tabular}

Abbreviations: GDM Gestational Diabetes Mellitus by IADPSG criteria, LGA Large for Gestational Age Delivery defined as $\geq 90$ th customized birthweight centile [26]. Customized centiles are adjusted for maternal age, height, weight, ethnic group, gestational age and gender. Weight adjustment for women with $\mathrm{BMI} \geq 30 \mathrm{Kg} / \mathrm{m}^{2}$ (all women in study) is based on a notional weight corresponding to a BMl of $29.9 \mathrm{Kg} / \mathrm{m}^{2}$.

Continuous variables are given as mean (SD) with Mean Difference \& 950025 Cl. Binary outcomes are $\mathrm{n}$ (\%), with Risk Difference and risk ratio. 
low levels of self efficacy. However, barriers to increasing PA appeared too great to overcome, possibly reflecting increased physical discomfort with gestation, as indicated by the EQ-5D questionnaire.

The relationship between mental health, diet and PA in obese pregnancies warrants further investigation in the RCT in view of the high prevalence of depressive symptoms (EPDS score $>12$ ). Another report has also found no effect of a complex behavioural intervention in obese women on these symptoms [60].

In terms of context, the process evaluation recruited women in urban hospitals serving regions with areas of high socio-economic deprivation. Obesity rates are higher amongst women with lower socio-economic status, fewer qualifications [61] and amongst particular ethnic groups, particularly black African and black Caribbean [62]. It was important therefore not only to explore if recruitment was feasible but also whether the intervention was acceptable to the women recruited. Prominent media coverage about obesity raised the possibility that women in the control group might proactively address diet and physical activity, and some interview data supported this, but the evaluation suggested that awareness through the media alone was not adequate to achieve sufficient behavioural change.

In relation to reach, just over one third of eligible women agreed to participate. Similarly low recruitment rates are consistent with other intervention studies, particularly in populations with lower uptake of health care. In one previous relevant study of lifestyle advice in nonobese pregnant women, recruitment was slower than expected and low attendance at group exercise sessions and participant concern about burdensome data collection contributed to dropout [63]. However, perceived advantages to participation such as extra clinical tests and continuity of care from research midwives supported study uptake and continuation. Given the continued rise in obesity in the adult population in England [61], approximately 1:5 pregnant women would be eligible for inclusion. Recruitment of the numbers needed to be approached (4100) for the full trial is therefore unlikely to be affected by a shortage of eligible women. Overall, the wide social and ethnic diversity amongst participants was similar in participants and those who declined, indicating that the intervention would be unlikely to increase health inequalities by attracting more educated and higher income participants [64]. Importantly, although obese pregnant women, once recruited, were generally willing to attend group sessions, practicalities often interfered with regular attendance, thereby influencing dose. However, the sessions did not appeal to all women. Some appreciated finding common issues with other group members, others preferred one to one contact. Evidence for health improvement interventions in group settings is varied [65] and this study adds to the recognition that a 'one size fits all' approach may not be effective [64], and that flexibility is key to retention. Fidelity was good with consistently high level provision of SMART goals by HTs, which were viewed as a positive achievement, particularly since poor adherence to goal setting has been associated with moderate attendance amongst pregnant women [66]. The high acceptability of the participant handbook and pedometer re-enforced the theoretical approach [16], and women also responded well to motivational techniques, but physical issues presented barriers to PA. The information provided was valued, including increasing awareness of safe PA in pregnancy, and seen to have important educational benefit. Several components of the intervention therefore appeared beneficial and were well received by women. The intervention is relatively intensive and presents costs for providers, and whilst a full assessment of cost and benefit was not conducted in this pilot, steps taken during the pre-clinical development phase (using HTs rather than clinicians to deliver the intervention, adopting local group-based approach) helped keep the overall costs of the intervention low, recognising that if beneficial, it should also be affordable to health providers and to women. There was also suggestion that the intervention may extend to peers and family, and some women aspired towards better fitness following birth. This study has reinforced earlier reports suggesting that rapport between study staff and participants, interviews requiring short time commitment, and participants' perception of the study as informative are all important recruitment and retention factors [67]. Formal evaluation of the reasons for the high refusal rate was not permissible due to ethical constraints, but the time commitment was frequently commented upon by the recruitment staff, as well as lack of appreciation of the health consequences of obesity in pregnancy.

In summary, this study has emphasised the value of a pilot trial to assess anticipated behaviour change. Although seldom attempted by others, we have also highlighted the importance of process evaluation in a complex intervention of diet and physical activity for pregnant women. The pilot trial demonstrated reductions in glycaemic load and in the proportion of energy derived from saturated fat are achievable in obese pregnant women without GDM. The process evaluation identified that dietary advice and education were well received, and confirmed that PA change is more problematic to achieve, although it remains important to consistently measure and support PA using technologies acceptable to women. The process evaluation also helped explain issues arising in relation to uptake, dose, fidelity and retention which informed the feasibility of the full trial. 
As a consequence of this study, several modifications to improve compliance and fidelity have now been implemented in the protocol for the main trial. As well as process evaluation, HT feedback highlighted potential barriers to fidelity of the intervention and informed protocol modifications for the RCT. Flexibility has been increased regarding the timing and delivery of the sessions, and goal setting can be undertaken by telephone or email. It is recommended that women should receive at least $5 / 8$ sessions. The two extra visits required for objective assessment of PA and accurate evaluation of diet have been omitted in all but two sites (as planned) and dietary assessment reduced to a validated FFQ. The RPAQ includes domestic and childcare activities, considered appropriate for pregnant women [68], but following feedback has been replaced by the shorter and more relevant IPAQ [69]. Maintenance of dietary and PA behaviour change in the participant and her family is being formally evaluated at 6 months and 3 years postpartum. To minimise loss to follow-up and attendance at these appointments, strategies which have been put in place include regular newsletters and sending greetings cards on special occasions such as the child's birthday.

\section{Conclusions}

Assumptions should not be made that interventions in obese pregnant women necessarily change behaviour. We recommend that a pilot trial such as that described here, which has demonstrated evidence for anticipated change in behaviour, is a necessary prelude to any RCT of a complex intervention of diet and physical activity designed to improve pregnancy outcome in obese women. Without prior evidence for change in behaviour in the anticipated direction, pursuit of a large and costly trial would be futile. Similarly, we have demonstrated the value of early process evaluation, which can lead to important refinements in protocol to improve feasibility and compliance in the definitive trial.

\section{Additional file}

Additional file 1: Table S1. Summary of session content; Table S2 Process intervention findings; Table S3, Structured interview sample characteristics; Table S4, Evaluation of intervention components.

Competing interests

The authors declare that they have no competing interests.

\section{Authors' contributions}

LP, ALB, RB, HC, NK, TK, TABS, JS, LMH EO-N and JW conceived the study, participated in its design and coordination and helped to draft the manuscript. SB, HC, KC, CH, LH, HNE and PTS performed the analyses. SB, HC, LMH, LH, NK, TK, SMN, EO-N, LP, NS, SCR, JW, TABS, JS helped to draft the manuscript. All authors read and approved the final manuscript.

\section{Acknowledgements}

We thank the study research midwives and the health trainers and all the pregnant women who took part.

This paper presents independent research commissioned by the National Institute for Health Research (NIHR) (UK) under the Programme Grants for Applied Research programme RP-0407-10452. The views expressed in this paper are those of the author(s) and not necessarily those of the Nationa Health Service, the NIHR or the Department of Health. The study was also supported by Guys and St.Thomas' Charity; Reg Charity 251983, UK; Chief Scientist Office, Scottish Government Health Directorates, Edinburgh, UK and Tommy's Charity; Reg Charity 1060508, UK.

\section{Author details}

'Division of Women's Health, Women's Health Academic Centre, King's College London and King's Health Partners, 10th floor, North Wing, St. Thomas' Hospital, London SE1 7EH, UK. Division of Diabetes and Nutritional Sciences, King's College London and King's Health Partners, London, UK. ${ }^{3}$ Institute of Health \& Society, Newcastle University, Newcastle, UK. Institute of Cellular Medicine, Newcastle University, Newcastle, UK. ${ }^{5}$ Department of Health Sciences, University of York, Newcastle, UK. ${ }^{6}$ Epidemiology and Public Health, University College London, London, UK. ${ }^{7}$ School of Medicine, University of Glasgow, Glasgow, UK. ${ }^{8}$ School of Health Sciences, University of Tampere, Tampere, Finland.

Received: 7 March 2013 Accepted: 7 July 2013

Published: 15 July 2013

\section{References}

1. World Health Organisation: WHO global info database. Data for saving lives. Internet: https://apps.who.int/infobase/ (accessed July 16 $6^{\text {th }}$ 2013).

2. Heslehurst N: Identifying 'at risk' women and the impact of maternal obesity on national health service maternity services. Proc Nutr Soc 2011, 70:439-449.

3. IOM (Institute of Medicine) and NRC (National Research Council): Weight Gain During Pregnancy: Reexamining the Guidelines. Washington, DC: The National Academies Press; 2009.

4. Thangaratinam S, Rogozinska E, Jolly K, Glinkowski S, Roseboom T, Tomlinson JW, Kunz R, Mol BW, Coomarasamy A, Khan KS: Effects of interventions in pregnancy on maternal weight and obstetric outcomes: meta-analysis of randomised evidence. BMJ 2012, 344:e2088.

5. Oteng-Ntim E, Varma R, Croker H, Poston L, Doyle P: Lifestyle interventions for overweight and obese pregnant women to improve pregnancy outcome: systematic review and meta-analysis. BMC Med 2012, 10:47.

6. Gardner B, Wardle J, Poston L, Croker H: Changing diet and physical activity to reduce gestational weight gain: a meta-analysis. Obesity Reviews 2011, 12:e602-e620.

7. Nelson S, Matthews P, Poston L: Maternal metabolism and obesity: modifiable determinants of pregnancy outcome. Hum Reprod Update 2010, 16:255-275.

8. Louie J, Brand-Miller JC, Moses RG: Carbohydrates, glycemic index, and pregnancy outcomes in gestational diabetes. Curr Diab Rep 2013, 13:6-11.

9. Tobias D, Zhang C, van Dam RM, Bowers K, Hu FB: Physical activity before and during pregnancy and risk of gestational diabetes mellitus: a metaanalysis. Diabetes Care 2011, 34:223-229.

10. Craig P, Dieppe P, Macintyre S, Michie S, Nazareth I, Petticrew M: Developing and evaluating complex interventions: the new medical research council guidance. BMJ 2008, 337:a1655.

11. Oteng-Ntim E, Pheasant H, Khazaezadeh N, Mohidden A, Bewley S, Wong J, Oke B: Developing a community-based maternal obesity intervention: a qualitative study of service providers' views. Br J Obstet Gynaecol 2010, 117:1651-1655.

12. Gardner B, Croker H, Barr S, Briley A, Poston L, Wardle J, UPBEAT Trial: Psychological predictors of dietary intentions in pregnancy. $J$ Hum Nutr Diet 2012, 25(4):345-353.

13. English Indices of Deprivation; 2010. Internet: https://www.gov.uk/ government/publications/english-indices-of-deprivation-2010 (accessed July $16^{\text {th }}$ 2013)

14. Scottish Indices of Multiple Deprivation; 2009. Internet: http://www.scotland. gov.uk/Publications/2009/10/28104046/0 (accessed July 16 ${ }^{\text {th }} 2013$ ).

15. National Institute for Health and Clinical Excellence: Dietary interventions and physical activity interventions for weight management before, during and after 
pregnancy; 2010. NICE PH27. Internet: http://guidance.nice.org.uk/PH27/ Guidance/pdf/English (accessed July 16th 2013).

16. Carver $\mathrm{C}$, Scheier M: Origins and functions of positive and negative affect: a control-process view. Psychological Review 1990, 97:19-35.

17. Bandura A: Health promotion by social cognitive means. Health Educ Behav 2004, 31:143-164.

18. Michie S, Abraham C, Whittington C, McAteer J, Gupta S: Effective techniques in healthy eating and physical activity interventions: a metaregression. Health Psychol 2009, 28:690-701.

19. Royal College of Obstetrics and Gynaecology: Exercise in pregnancy. Statement No. 4; 2006. Internet: http://www.rcog.org.uk/womens-health/ clinical-guidance/exercise-pregnancy (accessed July $16^{\text {th }}, 2013$ ).

20. Sechrist KR, Walker S, Pender NJ: Development and psychometric evaluation of the exercise benefits/barriers scale. Res Nurs Health 1987, 10:357-365.

21. Tiedje LB, Kingry MJ, Stommel M: Patient attitudes concerning health behaviors during pregnancy: initial development of a questionnaire. Health Educ Q 1992, 19:481-493.

22. The EuroQol Group: EuroQol-a new facility for the measurement of health-related quality of life. 1990, 16:199-208. Health Policy. Internet: http://www.euroqol.org (accessed $16^{\text {th }}$ July 2013).

23. Cox JL, Holden JM, Sagovsky R: Detection of postnatal depression. Development of the 10-item Edinburgh postnatal depression scale. $\mathrm{Br} J$ Psych 1987, 150:782-786.

24. Steckler A, Linnan L: Process evaluation for public health interventions and research. San Francisco, CA, US: Jossey-Bass. xxviii 400 pp 2002.

25. Metzger B, Gabbe SG, Persson B, Buchanan TA, Catalano PA, Damm P, Dyer AR, Leiva A, Hod M, Kitzmiler JL, Lowe LP, McIntyre HD, Oats JJ, Omori Y, Schmidt MI: International association of diabetes and pregnancy study groups recommendations on the diagnosis and classification of hyperglycemia in pregnancy. Diabetes Care 2010, 33:676-682.

26. Gardosi J, Francis A: Customised weight centile calculator - GROW - centile v5.15/6.4. Gestational Network 2009. Internet: http://www.gestation.net/ birthweight_centiles/online/centile_online_uk.htm (accessed 10th July, 2013).

27. Dolan P: Modeling valuations for EuroQol health states. Med Care 1997, 35:1095-1108

28. Dolan P, Gudex C, Kind P, Williams A: The time trade-off method: results from a general population study. Health Econ 1996, 5:141-154

29. Atkinson FS, Foster-Powell K, Brand-Miller JC: International tables of glycemic index and glycemic load values: 2008. Diabetes Care 2008, 31:2281-2283.

30. Aston LM, Jackson D, Monsheimer S, Whybrow S, Handjieva-Darlenska T, Kreutzer M, Kohl A, Papadaki A, Martinez JA, Kunova V, van Baak MA, Astrup A, Saris WH, Jebb SA, Lindroos AK: Developing a methodology for assigning glycaemic index values to foods consumed across Europe. Obes Rev 2010, 11:92-100.

31. Medical Research Council: Epidemiology Unit; MAHUffe program; 2012. Internet: http://www.mrc-epid.cam.ac.uk/research/resources/materialstransfer-disclaimer/physical-activity-downloads/ (accessed July 15 $5^{\text {th }}$ 2013).

32. Freedson PS, Melanson E, Sirard J: Calibration of the computer science and applications, Inc. accelerometer. Med Sci Sports Exerc 1998, 30:777-781.

33. Matthew CE: Calibration of accelerometer output for adults. Med Sci Sports Exerc 2005, 37(11 Suppl):S512-S522.

34. Ainsworth BE, Haskell WL, Whitt MC, Irwin ML, Swartz AM, Strath SJ, O'Brien WL, Bassett DR Jr, Schmitz KH, Emplaincourt PO, Jacobs DR Jr, Leon AS: Compendium of physical activities: an update of activity codes and MET intensities. Med Sci Sports Exerc 2000, 32(9 Suppl):S498-S504.

35. Green J, Thorogood N: Qualitative Methods for Health Research 2. London: Ed Sage; 2009.

36. Centre for Maternal and Child Enquiries and the Royal College of Obstetricians and Gynaecologists Joint Guideline: Management of women with obesity in pregnancy; 2010. Internet; http://www.rcog.org.uk/womenshealth/clinical-guidance/management-women-obesity-pregnancy (accessed July 10th 2013).

37. Quinlivan JA, Lam LT, Fisher J: A randomised trial of a four-step multidisciplinary approach to the antenatal care of obese pregnant women. Aust N Z J Obstet Gynaecol 2011, 51:141-146.

38. Guelinckx I, Devlieger R, Mullie P, Vansant G: Effect of lifestyle intervention on dietary habits, physical activity, and gestational weight gain in obese pregnant women: a randomized controlled trial. Am J Clin Nutr 2010, 91:373-380.

39. Bogaerts A, Devlieger R, Nuyts E, Witters I, Gyselaers W, Van den Bergh BR: Effects of lifestyle intervention in obese pregnant women on gestational weight gain and mental health: a randomized controlled trial. Int J Obes (Lond) 2012. Epub ahead of print.

40. Whitton C, Nicholson SK, Roberts C, Prynne CJ, Pot GK, Olson A, Fitt E, Cole D, Teucher B, Bates B, Henderson H, Pigott S, Deverill C, Swan G, Stephen AM: National diet and nutrition survey: UK food consumption and nutrient intakes from the first year of the rolling programme and comparisons with previous surveys. Br J Nutr 2011, 106:1899-1914.

41. Fabricatore AN, Wadden TA, Ebbeling CB, Thomas JG, Stallings VA, Schwartz S, Ludwig DS: Targeting dietary fat or glycemic load in the treatment of obesity and type 2 diabetes: a randomized controlled trial. Diabetes Res Clin Pract 2011, 92:37-45.

42. Walsh JM, McGowan CA, Mahony R, Foley ME, McAuliffe FM: Low glycaemic index diet in pregnancy to prevent macrosomia (ROLO study): randomised control trial. BMJ 2012, 345:e5605.

43. Jebb SA, Lovegrove JA, Griffin BA, Frost GS, Moore CS, Chatfield MD, Bluck LJ, Williams CM, Sanders TA, RISCK Study Group: Effect of changing the amount and type of fat and carbohydrate on insulin sensitivity and cardiovascular risk: the RISCK (Reading, Imperial, Surrey, Cambridge, and Kings) Trial. Am J Clin Nutr 2010, 92:748-758.

44. Thomas DE, Elliott EJ, Baur L: Low glycaemic index or low glycaemic load diets for overweight and obesity. Cochrane Database Syst Rev 2007, 3, CD005105.

45. Department of Health: Dietary Reference Values for Food Energy and Nutrients for the United Kingdom. Report on Health and Social Subjects 41. HSMO, London: The Stationary Office; 1991.

46. Hui A, Back L, Ludwig S, Gardiner P, Sevenhuysen G, Dean H, Sellers E, McGavock J, Morris M, Bruce S, Murray R, Shen GX: Lifestyle intervention on diet and exercise reduced excessive gestational weight gain in pregnant women under a randomised controlled trial. Br J Obstet Gynaecol 2012, 119:70-77.

47. Callaway LK, Colditz PB, Byrne NM, Lingwood BE, Rowlands IJ, Foxcroft K, Mclntyre HD, BAMBINO Group: Prevention of gestational diabetes: feasibility issues for an exercise intervention in obese pregnant women. Diabetes Care 2010, 33:1457-1459.

48. Evenson KR, Wen F: Prevalence and correlates of objectively measured physical activity and sedentary behavior among US pregnant women. Prev Med 2011, 53:39-43.

49. Aittasalo M, Raitanen J, Kinnunen TI, Ojala K, Kolu P, Luoto R: Is intensive counseling in maternity care feasible and effective in promoting physical activity among women at risk for gestational diabetes? Secondary analysis of a cluster randomized NELLI study in Finland. Int J Behav Nutr Phys Act 2012, 9:104.

50. Oostdam N, van Poppel MN, Wouters MG, Eekhoff EM, Bekedam DJ, Kuchenbecker WK, Quartero HW, Heres MH, van Mechelen W: No effect of the FitFor2 exercise programme on blood glucose, insulin sensitivity, and birthweight in pregnant women who were overweight and at risk for gestational diabetes: results of a randomised controlled trial. $\mathrm{Br} J$ Obstet Gynecol 2012, 119:1098-1107.

51. Prince SA, Adamo KB, Hamel ME, Hardt J, Gorber SC, Tremblay M: A comparison of direct versus self-report measures for assessing physical activity in adults: a systematic review. Int J Behav Nutr Phys Act 2008, 5:56.

52. Schmidt MD, Freedson PS, Pekow P, Roberts D, Sternfeld B, Chasan-Taber L: Validation of the kaiser physical activity survey in pregnant women. Med Sci Sports Exerc 2006, 38:42-50.

53. McParlin C, Robson SC, Tennant PW, Besson H, Rankin J, Adamson AJ, Pearce MS, Bell R: Objectively measured physical activity during pregnancy: a study in obese and overweight women. BMC Pregnancy Childbirth 2010, 10:76.

54. Rousham EK, Clarke PE, Gross H: Significant changes in physical activity among pregnant women in the UK as assessed by accelerometry and self-reported activity. Eur J Clin Nutr 2006, 60:393-400.

55. Evenson KR, Savitz DA, Huston SL: Leisure-time physical activity among pregnant women in the US. Paediatr Perinat Epidemiol 2004, 18:400-407.

56. Townsend N, Bhatnagar P, Wickramasinghe K, Scarborough P, Foster C, Rayner M: Physical activity statistics 2012. London: British Heart Foundation; 2012.

57. Stein AD, Rivera JM, Pivarnik JM: Measuring energy expenditure in habitually active and sedentary pregnant women. Med Sci Sports Exerc 2003, 35:1441-1446.

58. Lof M, Forsum E: Activity pattern and energy expenditure due to physical activity before and during pregnancy in healthy Swedish women. $\mathrm{Br} J$ Nutr 2006, 95:296-302. 
59. Harrison $\mathrm{CL}$, Lombard $\mathrm{CB}$, Teede $\mathrm{HJ}$ : Understanding health behaviours in a cohort of pregnant women at risk of gestational diabetes mellitus: an observational study. Br J Obstet Gynaecol 2012, 119:731-738.

60. Claesson IM, Josefsson A, Sydsjo G: Prevalence of anxiety and depressive symptoms among obese pregnant and postpartum women: an intervention study. BMC Public Health 2010, 10:766.

61. Health and Social Care Information Centre: Statistics on obesity, physical activity and diet: England; 2013. Internet: http://www.hscic.gov.uk/catalogue/ PUB10364 (accessed July 15 ${ }^{\text {th }}$ 2013).

62. Gatineau M, Mathrani S: Public Health England. Obesity and Ethnicity, Oxford: National Obesity Observatory 2011. Internet: http://www.noo.org.uk/ NOO_pub/briefing_papers.

63. Kinnunen $\mathrm{Tl}$, Aittasalo M, Koponen P, Ojala K, Mansikkamaki K, Weiderpass E, Fogelholm M, Luoto R: Feasibility of a controlled trial aiming to prevent excessive pregnancy-related weight gain in primary health care. $B M C$ Pregnancy Childbirth 2008, 8:37.

64. Hoddinott P, Allan K, Avenell A, Britten J: Group interventions to improve health outcomes: a framework for their design and delivery. BMC Public Health 2010, 10:800.

65. National Institute for Health and Clinical Excellence: The most appropriate means of generic and specific interventions to support attitude and behaviour change at population and community levels. Public health guidance, PH6 2007. Internet: http://publications.nice.org.uk/behaviourchange-ph6 (accessed 10 $0^{\text {th }}$ July 2013).

66. Broekhuizen K, Althuizen E, van Poppel MN, Donker M, van Mechelen W: From theory to practice: intervention fidelity in a randomized controlled trial aiming to optimize weight development during pregnancy. Health Promot Pract 2012, 13:816-825.

67. Barnett J, Aguilar S, Brittner M, Bonuck K: Recruiting and retaining lowincome, multi-ethnic women into randomized controlled trials: successful strategies and staffing. Contemp Clin Trials 2012, 33:925-932.

68. Borodulin K, Evenson KR, Herring AH: Physical activity patterns during pregnancy through postpartum. BMC Womens Health 2009, 9:32.

69. Hallal P, Victora CG: Reliability and validity of the International Physical Activity Questionnaire (IPAQ). Med Sci Sports Exerc 2004, 36:556.

doi:10.1186/1471-2393-13-148

Cite this article as: Poston et al:: Developing a complex intervention for diet and activity behaviour change in obese pregnant women (the UPBEAT trial); assessment of behavioural change and process evaluation in a pilot randomised controlled trial. BMC Pregnancy and Childbirth 2013 13:148.

\section{Submit your next manuscript to BioMed Central and take full advantage of:}

- Convenient online submission

- Thorough peer review

- No space constraints or color figure charges

- Immediate publication on acceptance

- Inclusion in PubMed, CAS, Scopus and Google Scholar

- Research which is freely available for redistribution 